\title{
MAPA DE CONVERSAÇÃO COMO ESTRATÉGIA DE ENSINO PARA O DESENVOLVIMENTO DO AUTOCUIDADO EM DIABETES MELLITUS
}

Rosali Isabel Barduchi Ohl'1, Suzel Regina Ribeiro Chavaglia ${ }^{2}$, Julianna Leticia Gimenes Cotrick Gomes ${ }^{1}$, Maria Aparecida de Oliveira Freitas ${ }^{1}$ Mônica Antar Gamba ${ }^{1}$

Objetivo: Avaliar o Mapa de Conversação como estratégia de ensino para o desenvolvimento do autocuidado de pessoas diabéticas. Medtodologia: Estudo descritivo correlacional com abordagem quantitativa. Participaram do programa educativo, 20 usuários. Resultados: O período de execução do programa foi de oito semanas. A avaliação das ações educativas foi testada pela aplicação do Questionário de Atividades para o Autocuidado em Diabetes, cujos escores foram analisados no início e no final do programa. A variável 'ressecamento', relativa às características da pele e as variáveis "Alimentação específica (doces)", "atividade física", "glicemia capilar" e "cuidado com os pés" apresentaram significância estatística (p<0,05). Conclusão: Programas educativos são essenciais para a capacitação da pessoa diabética e a mudança de seu comportamento em relação ao seu autocuidado. O Mapa de Conversação em Diabetes demonstrou ser adequado para aquisição de novos conhecimentos e autonomia da pessoa no controle glicêmico.

Descritores: Diabetes mellitus; Educação em saúde; Autocuidado; Enfermagem, Estratégia de Saúde da Família.

\section{CONVERSATION MAP AS A TEACHING STRATEGY FOR THE DEVELOPMENT OF SELF-CARE IN DIABETES MELLITUS ABSTRACT}

Objective: To evaluate the Conversation Map as a teaching strategy for the development of self-care for diabetic people. Methodology: Descriptive correlational study with quantitative approach. Twenty users participated in the educational program. Results: The period of execution of the program was eight weeks. The assessment of educational actions was tested by the application of the Diabetes Self-Care Activity Questionnaire (DSCAQ), whose scores were analyzed at the beginning and at the end of the program. The "dryness" variable, related to the characteristics of the skin and the variables "Specific diet (sweets)", "physical activity", "capillary glycemia" and "foot care" were statistically significant ( $p<0.05)$. Conclusion: Educational programs are essential for the empowerment of the diabetic person and the change in their behavior regarding self-care. The Conversation Map demonstrated to be adequate for the acquisition of new knowledge and autonomy of the person in glycemic control.

Descriptors: Diabetes mellitus; Health education; Self-care; Nursing; Family Health Strategy.

\section{MAPA DE LA CONVERSACIÓN COMO ESTRATEGIA DE ENSEÑANZA PARA EL DESARROLLO DEL AUTO CUIDADO EN DIABETES MELLITUS}

Objetivo: Evaluar el Mapa de Conversación como estrategia de enseñanza para el desarrollo del auto cuidado de personas diabéticas. Medodología: Estudio descriptivo correlacional con enfoque cuantitativo. Participaron del programa educativo, 20 usuarios. Resultados: El período de ejecución del programa fue de ocho semanas. La evaluación de las acciones educativas fue testada por la aplicación del Cuestionario de Actividades para el Autocuidado en Diabetes (CAAD), cuyos escores se analizaron al principio y al final del programa. La variable "resecamiento", relativa a las características de la piel y las variables "Alimentación específica (dulces)", "actividad física", "glucemia capilar" y "cuidado con los pies" presentaron significancia estadística (p $<0,05)$. Conclusión: Los programas educativos son esenciales para la capacitación de la persona diabética y el cambio de su comportamiento en relación al auto cuidado. El Mapa de Conversación demostró ser adecuado para la adquisición de nuevos conocimientos y autonomía de la persona en el control glucémico.

Descriptores: Diabetes Mellitus; Educación en salud; Autocuidado; Enfermería; Estrategia de Salud Familiar. 


\section{INTRODUÇÃO}

O Diabetes Mellitus (DM) é uma doença crônica que exige mudanças de hábitos e desenvolvimento de comportamentos especiais de autocuidado que deverão ser mantidos por toda a vida. A educação em DM deve promover o desenvolvimento de habilidades necessárias para o autocuidado e para o gerenciamento da doença pela própria pessoa e/ou familiar. Só podemos considerar a educação em DM efetiva se ela resultar em mudanças e/ou incorporação de novos comportamentos cotidianos ${ }^{(1,2)}$.

O Ministério da Saúde tem estruturado importantes ações de atenção para a prevenção e o controle das Doenças Crônicas Não Transmissiveis - DCNT, dentre elas o DM, seus fatores de risco e fortalecimento dos serviços de saúde, objetivando o desenvolvimento e a implementação de políticas públicas efetivas, integradas, sustentáveis e baseadas em evidências ${ }^{(3,4)}$. Essas ações educativas junto às pessoas com DM devem ir além de imprimir conhecimentos científicos, mas sim, envolver aspectos emocionais e socioculturais que visem contribuir no reconhecimento de suas condições de saúde, proporcionar as transformações necessárias em seus estilos de vida e, assim, influenciar na adesão ao tratamento(5).

A estratégia de se trabalhar em grupos educativos pode facilitar o desenvolvimento de novos saberes e práticas que levam ao fortalecimento de vínculo entre a equipe de saúde e o cliente, o que faz com que ele se sinta acolhido e corresponsável para aderir e realizar o tratamento ${ }^{(6,7)}$. Assim, deve-se buscar a melhor abordagem para orientar e efetivamente preparar a pessoa com DM, para que ela seja protagonista do seu tratamento e da sua saúde. Prepará-la para que saiba o que fazer com os resultados dos seus exames laboratoriais, reconheça os seus sinais e sintomas e tenha um enfrentamento saudável da doença.

O Mapa de Conversação em Diabetes ${ }^{\circledR(8)}$ tem sido utilizado como estratégia de ensino para o alcance do autocuidado em diversos países. Ele tem sido utilizado em diversos estudos randomizados em países como Nigéria, China e Brasil, proporcionando o alivio do sofrimento, aumento da autoeficácia, e melhora da Hemoglobina glicada (HAlc) na população diabética, comparando-se ao ensino tradicional realizado junto à pacientes com DM Tipo 2 (DM2)(9-11).

\section{OBJETIVO}

Avaliar o Mapa de Conversação em Diabetes ${ }^{\circledR}$ como estratégia de ensino facilitadora do desenvolvimento do autocuidado de pessoas diabéticas cadastradas em uma unidade de Estratégia de Saúde da Família.

\section{METODOLOGIA}

\section{Tipo de estudo}

Estudo descritivo correlacional( ${ }^{(12)}$ sobre educação em DM na atenção primária à saúde.

\section{Participantes da Pesquisa}

Foram convidados 122 usuários cadastrados junto às equipes deESF, com diagnóstico da ClassificaçãoInternacional de Doenças - CIDIO: ElO (DM insulinodependente) e El (DM não-insulinodependente), porém participaram de todas as etapas do programa educativo somente 20 usuários, distribuídos em dois grupos de 10 pessoas para a aplicação da estratégia de ensino.

Os critérios de inclusão foram: pessoas cadastradas na ESF com diagnóstico de DM que consentiram em sua participar do estudo após assinatura do Termo de Consentimento Livre e Esclarecido. Foram excluídos aqueles usuários com quadros demenciais aparentes e acamados.

\section{Local de Estudo}

A pesquisa foi desenvolvida no periodo de abril a julho de 2014 em uma área da Estratégia de Saúde da Família - ESF pertencente a uma Unidade Básica de Saúde (UBS) de um município de São Paulo - SP.

\section{Coleta de Dados - Protocolo do estudo}

O programa educativo foi desenvolvido por meio do Mapa de Conversação em Diabetes®(8) (Figura 1), com atividades semanais, realizadas durante oito semanas consecutivas e temas baseados nas necessidades individuais e coletivas dos participantes.

Figura 1. Mapa de Conversação em Diabetes ${ }^{\circledR}$ - Tela: O Diabetes e o Cuidado de seus Pés. Ribeirão Preto - SP, 2018.

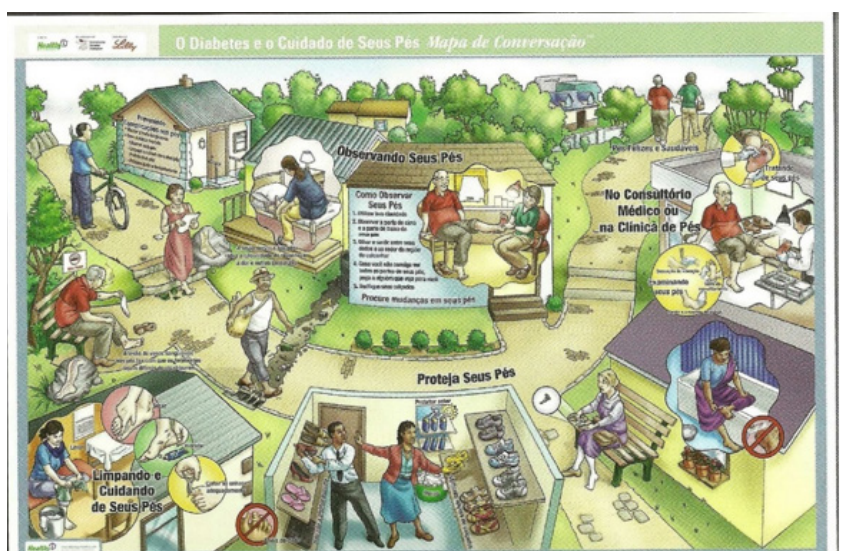

Fonte: Prefeitura Municipal de Ribeirão Preto, SP. 2018 Disponivel em: http://www.ribeiraopreto.sp.gov.br/ssaude/ pdf/05_diabetes_seus_pes.pdf 
O Mapa de Conversação em Diabetes® foi planejado e desenvolvido segundo os conteúdos estabelecidos nas diretrizes de educação em DM propostas pela American Association of Diabetes Educators (AADE) e Sociedade Brasileira de Diabetes - SBD, tendo como base os sete comportamentos de autocuidado que devem ser praticados pelos pacientes após realização de trabalho educativo efetivo: Comendo saudavelmente; Fazendo Atividade Física; Vigiando as taxas; Tomando os medicamentos; Encontrando soluções; Reduzindo os riscos; Adaptando-se saudavelmente.

\section{Procedimento de Análise dos Dados}

Os dados foram elaborados através do programa estatístico Epi Info versão 3.3.2, realizando-se codificação das variáveis transcritas para esse programa. Os bancos de dados eletrônicos foram preparados de maneira a permitir sua posterior comparação da dupla digitação. Os dados foram apresentados por estatística descritiva através de tabelas.

As variáveis sociodemográficas avaliadas foram: idade, sexo, cor da pele, escolaridade, estado civil, renda. As variáveis clínicas e laboratoriais foram: níveis de glicemia em jejum, índices de HbAlc, perfil lipídico (HDL, LDL, Triglicérides); pressão arterial, Índice de Massa Corpórea - IMC, avaliação dos pés, hábitos e comportamentos para o autocuidado. As atividades de autocuidado antes e após o desenvolvimento do programa educativo foram avaliadas utilizando-se o Questionário de Atividades de Autocuidado com o Diabetes (OAD)(13). O alfa de Cronbach ( ) foi utilizado para avaliar a consistência interna e a confiabilidade do questionário, e a correlação interitens variou de $=0,09 \mathrm{a}=0,86$.

\section{Procedimentos éticos}

O projeto de pesquisa foi encaminhado e submetido à análise e aprovação do Comitê de Ética em Pesquisa (CEP) de uma instituição federal de ensino, sob número $\mathrm{n}$ 은 618.135, CAAE 24937214.1.0000.5505. As pessoas foram informadas sobre a pesquisa e seus objetivos, e incluídas no estudo, após assinatura do Termo de Consentimento Livre (TCL) para participação, assegurando-se o anonimato e a confidencialidade dos dados.

\section{RESULTADOS}

A maioria dos 20 sujeitos que aceitaram participar e concluíram o programa são do sexo feminino (70\%), idade média de 59,6 anos, cor branca (65\%), baixo nível de escolaridade (60\%), utilizavam exclusivamente o Sistema Único de Saúde - SUS (90\%), antidiabético oral (75\%), obesos, IMC média de $31 \mathrm{~kg} / \mathrm{m} 2$, média de circunferência abdominal de $104 \mathrm{~cm}$. A variação média da pressão arterial sistólica e diastólica não foi significativa estatisticamente entre os periodos de observação.

No item hidratação da pele dos membros inferiores, evidenciou-se ressecamento em 17 (85\%) pessoas no início do programa e dessas, 06 (30\%) pessoas apresentaram pele hidratada ao final do programa, com diferença estatisticamente significativa $(p=0,041)$ (Tabela 1$)$.

Tabela 1. Alterações identificadas na pele e anexos dos membros inferiores de pessoas com DM antes e depois do programa educativo. São Paulo - SP, 2018.

\begin{tabular}{|c|c|c|c|}
\hline Características da pele & Antes n (\%) & Depois $n(\%)$ & Valor de $\mathrm{Pl}^{1}$ \\
\hline \multicolumn{4}{|c|}{ Alteração na sensibilidade protetora } \\
\hline $\operatorname{Sim} n(\%)$ & $3(12,0)$ & $1(6,0)$ & $\sim 1,000$ \\
\hline Não n (\%) & $16(84,0)$ & $18(94,0)$ & \\
\hline \multicolumn{4}{|l|}{ Ressecamento } \\
\hline $\operatorname{Sim} n(\%)$ & $11(57,0)$ & $6(32,0)$ & 0,041 \\
\hline Não n (\%) & $8(43,0)$ & $13(68,0)$ & \\
\hline \multicolumn{4}{|l|}{ Onicomicose } \\
\hline $\operatorname{Sim} n(\%)$ & $3(12,0)$ & $1(6,0)$ & $\sim 1,000$ \\
\hline Não n (\%) & $16(84,0)$ & $18(94,0)$ & \\
\hline \multicolumn{4}{|l|}{ Calosidade } \\
\hline $\operatorname{Sim} n(\%)$ & $4(21,0)$ & $1(6,0)$ & $\sim 1,000$ \\
\hline Não n (\%) & $15(79,0)$ & $18(94,0)$ & \\
\hline
\end{tabular}

${ }^{1}$ Teste de McNemar

A avaliação dos hábitos e comportamentos para o autocuidado foi testada pela aplicação do Questionário de Atividades para o Autocuidado em Diabetes (OAD), cujos escores foram analisados no início e no final do programa educativo. (Tabela 2) 
Tabela 2. Escores do QAD em relação às variáveis: alimentação geral e específica, atividade física, monitorização da glicemia, cuidado com os pés e uso das medicações prescritas antes e depois do projeto educativo. São Paulo - SP, 2018

\begin{tabular}{|c|c|c|c|c|c|c|c|}
\hline Varnavers & & Medıa & padrào & Mediana & $\mathbf{P}$ & antes & depois \\
\hline \multicolumn{8}{|l|}{ Alimentação Geral } \\
\hline Q1 - Em quantos dos últimos sete & Inicial & & 3,6 & 2,9 & 3,0 & $0,010^{*}$ & 0,69 \\
\hline dias seguiu uma dieta saudável? & Final & & 5,9 & 1,4 & 7,0 & & \\
\hline Q2 - Durante o último mês, & Inicial & & 1,9 & 2,9 & 0,0 & $<0,001^{*}$ & \\
\hline $\begin{array}{l}\text { quantos dias por semana, em } \\
\text { média, seguiu a orientaçấo }\end{array}$ & Final & & 5,6 & 2,1 & 7,0 & & \\
\hline \multicolumn{8}{|l|}{$\begin{array}{l}\text { alimentar dada por um } \\
\text { profissional de saúde? }\end{array}$} \\
\hline \multicolumn{8}{|l|}{ Alimentação Especifica } \\
\hline Q3-Em quantos dos últimos sete & Inicial & 3,7 & 3,2 & 5,0 & 0,370 & $-2,05$ & $-0,51$ \\
\hline $\begin{array}{l}\text { dias comeu cinco ou mais porçôes } \\
\text { de frutas e/ou vegetais? }\end{array}$ & Final & 4,9 & 2,4 & & & & \\
\hline Q4 - Em quantos dos últimos sete & Inicial & 4,1 & 3,0 & 5,0 & 0,420 & & \\
\hline $\begin{array}{l}\text { dias comeu alimentos ricos em } \\
\text { gordura, como carnes vermellhas }\end{array}$ & Final & 3,0 & 2,1 & 2,0 & & & \\
\hline \multicolumn{8}{|l|}{$\begin{array}{l}\text { ou alimentos com leite integral ou } \\
\text { derivados? }\end{array}$} \\
\hline Q5 - Em quantos dos últimos gete & Inicial & 2,2 & 1,9 & 2,0 & $0,018^{*}$ & & \\
\hline dias comeu doces? & Final & 0,9 & 0,9 & 1,0 & & & \\
\hline \multicolumn{8}{|l|}{ Atividade Fisica } \\
\hline Q6 - Em quantos dos últimos sete & Inicial & 1,4 & 2,3 & 0,0 & 0,150 & 0,31 & 0,76 \\
\hline $\begin{array}{l}\text { dias realizou atividades fíicas } \\
\text { durante pelo menos } 30 \text { minutos? }\end{array}$ & Final & 2,4 & 1,7 & 2,0 & & & \\
\hline \multicolumn{8}{|l|}{$\begin{array}{l}\text { (Contando com as atividades do } \\
\text { trabalho) }\end{array}$} \\
\hline Q7 - Em quantos dos últimos sete & Inicial & 0,3 & 0,9 & 0,0 & $<0,001^{*}$ & & \\
\hline $\begin{array}{l}\text { dias praticou algum tipo de } \\
\text { exercício fisico especifico, sem }\end{array}$ & Final & 2,3 & 1,7 & 2,0 & & & \\
\hline \multicolumn{8}{|l|}{$\begin{array}{l}\text { incluir suas atividades de } \\
\text { trabalho? }\end{array}$} \\
\hline \multicolumn{8}{|l|}{ Monitorização da Glicemia } \\
\hline Q8 - Em quantos dos últimos gete & Inicial & 1,3 & 1,7 & 1,0 & $0,007^{*}$ & 0,66 & 0,94 \\
\hline dias avaliou o açúcar no sangue? & Final & 3,1 & 2,4 & 2,0 & & & \\
\hline Q9- Em quantos dos últimos sete & Inicial & 0,7 & 1,1 & 0,0 & $<0,001^{*}$ & & \\
\hline $\begin{array}{l}\text { dias avaliou o açucar no sangue o } \\
\text { número de vezer recomendado }\end{array}$ & Final & 3,1 & 2,4 & 2,0 & & & \\
\hline \multirow{2}{*}{\multicolumn{8}{|c|}{$\begin{array}{l}\text { pelo médico ou enfermeiro? } \\
\text { Cuidado com os Pés }\end{array}$}} \\
\hline & & & & & & & \\
\hline Q10 - Em quantos dos últimos sete & Inicial & 2,6 & 3,2 & 1,0 & $<0,001^{*}$ & 0,24 & 0,47 \\
\hline dias examinou os seus pés? & Final & 6,1 & 2,1 & 7,0 & & & \\
\hline Q11 - Em quantos dos últimos sete & Inicial & 3,1 & 3,3 & 1,5 & $<0,001^{*}$ & & \\
\hline $\begin{array}{l}\text { dias examinou dentro dos sapatos } \\
\text { antes de calca-los? }\end{array}$ & Final & 6,6 & 1,6 & 7,0 & & & \\
\hline Q12 - Em quantos dos últimos & Inicial & 6,5 & 1,7 & 7,0 & 0,160 & & \\
\hline gete dias secou os espaços entre os & Final & 7,0 & 0,0 & 7,0 & & & \\
\hline \multirow{2}{*}{\multicolumn{8}{|c|}{$\begin{array}{l}\text { dedos dos pés depois de lavá-los? } \\
\text { Uso das Medicaçốes Prescritas }\end{array}$}} \\
\hline & & & & & & & \\
\hline Q13 - Em quantos dos últimos & Inicial & 6,6 & 0,7 & 7,0 & 0,17 & 0,79 & 0,00 \\
\hline sete dias tomou seus & Final & 6,9 & 0,2 & 7,0 & & & \\
\hline \multicolumn{8}{|l|}{$\begin{array}{l}\text { medicamentos do diabetes, } \\
\text { conforme foi recomendado? }\end{array}$} \\
\hline Q14- Em quantos dos últimos sete & Inicial & 4,2 & 3,8 & 7,0 & 0,48 & & \\
\hline $\begin{array}{l}\text { dias tomou suas injeçōes de } \\
\text { insulina, conforme foi } \\
\text { recomendado? }\end{array}$ & Final & 5,8 & 2,8 & 7,0 & & & \\
\hline Q15 - Em quantos dos últimos & Inicial & 6,0 & 2,2 & 7,0 & 0,08 & & \\
\hline $\begin{array}{l}\text { sete dias tomou o numero de } \\
\text { comprimidos do diabetes? }\end{array}$ & Final & 6,9 & 0,2 & 7,0 & & & \\
\hline
\end{tabular}

Fonte: Dados primários do Programa

$\mathrm{Na}$ dimensão alimentação geral, observou-se aumento significativo dos dias em que o participante respondeu seguir dieta saudável, com média de 3,6 dias antes do programa e posteriormente de 5,9 dias. Para o item ingesta de dieta orientada por um profissional da saúde, a média de dias aumentou de 1,9 dias para 5,6 dias após o programa educativo.

Quanto à alimentação específica, nota-se discreto aumento no número de dias de consumo de frutas e vegetais (3,7 para 4,9 dias). Já para o item diminuição do consumo de alimentos gordurosos, carne vermelha, leite integral e derivados, a média diminuiu de 4,1 para 3,0 dias; ingesta de doces de 2,2 para 0,9 dias, sendo que a diminuição de doces foi estatisticamente significativa $(p=0,018)$.

O aspecto "Atividade Física" revelou aumento do número de dias em que as pessoas realizaram exercício físico não atrelado às atividades de trabalho.
Quando interrogados sobre atividades físicas, considerando-se as atividades de trabalho durante pelo menos 30 minutos nos últimos sete dias, a média de dias na fase inicial foi de 1,4 e 2,4 dias no final. Na aferição destes dados sem as atividades de trabalho, a média de dias passou de 0,3 para 2,3 dias.

Em relação aos parâmetros clínicos, obteve-se diferença nos níveis da HbAlc e a glicemia de jejum antes e depois do programa. Para HbAlc a média inicial foi de $7,6 \%$ e a final de $6,6 \%$, obtida após o teste Estatístico de Wilcoxon ( $p=0.008)$, demonstrando diferença estatística significativa.

Tabela 3- Distribuição da média, desvio padrão, mediana e valor de $p$ relacionados aos parâmetros bioquímicos dos participantes do Programa Educativo, São Paulo, SP, 2018.

\begin{tabular}{|c|c|c|c|c|c|}
\hline Variáveis & Periodo & Média & $\begin{array}{l}\text { Desvio } \\
\text { padrão }\end{array}$ & Mediana & Valor de $\mathrm{P}]$ \\
\hline \multirow{2}{*}{$\begin{array}{l}\text { Hemoglobina } \\
\text { Glicada (\%) }\end{array}$} & Inicial & 7,6 & 1,6 & 7,5 & $0,008^{*}$ \\
\hline & Final & 6,6 & 1,2 & 6,7 & \\
\hline \multirow{2}{*}{$\begin{array}{l}\text { Glicemia de } \\
\text { jejum(mg/dl) }\end{array}$} & Inicial & 140,3 & 42,1 & 131,0 & 0,550 \\
\hline & Final & 135,2 & 48,8 & 130,0 & \\
\hline \multirow{2}{*}{$\begin{array}{l}\text { Colesterol total } \\
(\mathrm{mg} / \mathrm{dl})\end{array}$} & Inicial & 206 & 35 & 204 & 0,12 \\
\hline & Final & 187 & 31 & 187 & \\
\hline \multirow{2}{*}{$\mathrm{LDL}(\mathrm{mg} / \mathrm{dl})$} & Inicial & 125 & 29 & 119 & 0,24 \\
\hline & Final & 113 & 28 & 106 & \\
\hline \multirow{2}{*}{$\mathrm{HDL}(\mathrm{mg} / \mathrm{dl})$} & Inicial & 41 & 07 & 42 & 0,14 \\
\hline & Final & 39 & 09 & 40 & \\
\hline \multirow{2}{*}{$\begin{array}{l}\text { Triglicérides(mg/ } \\
\text { dl) }\end{array}$} & Inicial & 189 & 71 & 187 & 0,12 \\
\hline & Final & 172 & 63 & 165 & \\
\hline \multirow{2}{*}{$\begin{array}{l}\text { Microalbuminúria } \\
(\mu \mathrm{g} / \mathrm{min})\end{array}$} & Inicial & 58 & 116 & 15 & 0,45 \\
\hline & Final & 63 & 127 & 14 & \\
\hline
\end{tabular}

Fonte: Dados primários da pesquisa

${ }^{1}$ Teste pareado de Wilcoxon

\section{DISCUSSÃO}

Dentre os 122 usuários convidados, somente 20 (16,4,0\%) pessoas aceitaram participar do estudo, chegando ao seu término. A maior parte das desistências se relacionou à falta de tempo e/ou interesse dos sujeitos. Estudo semelhante realizado em um hospital no município de Belo Horizonte (MG) descreve participação menos efetiva nas atividades educativas, onde, dos 57 convidados, 30 pessoas (52,6\%) não compareceram e os motivos declarados foram de ordem econômica e institucional/empregatícia, dados que corroboram com os achados deste estudo(14).

Torna-se necessário a aplicação de estratégias educativas que estimulem a participação dos usuários nessas práticas. O enfermeiro deve, portanto, primeiramente, conhecer a realidade dos sujeitos e, posteriormente, em conjunto com eles visualizar possibilidades de transformações para 
uma maior resolutividade dos problemas de saúde desta população(15)

Estudos apontam que a educação em grupo se mostra mais efetiva quando comparada à educação individual, fato esse que pode ser explicado pela troca de experiências, suporte e apoio social(15,16). Pesquisa de revisão sistemática com metanálise de 21 estudos que avaliaram o efeito da educação em saúde realizada em grupo assegurou efeito positivo nos valores de $\mathrm{HbAlc}$, mantido em até 24 meses após a intervenção grupal ${ }^{(17)}$.

Já em outro estudo de metanálise cujo objetivo foi avaliar a eficácia da educação individual do paciente no controle metabólico, o conhecimento diabetes e resultados psicossociais demonstrou que a educação individual proporcionou maior conhecimento, maior autoeficácia, melhora significativa no controle glicêmico e redução do sofrimento relacionado ao $\mathrm{DM}^{(18)}$.

Investigações descritivas e de intervenção educativa em DM divulgam a experiência do uso da estratégia de ensinoaprendizagem mapa de conversação em diferentes países, como, Alemanha, Austrália, China, Nigéria, Reino Unido e Estados Unidos ${ }^{(10-12,19-21)}$ observando que a ferramenta facilitou o diálogo e interação entre os indivíduos com DM, gerando discussões e troca de experiências sobre o como é viver com a doença.

A literatura corrobora com as informações sociodemográficas observadas neste estudo, onde o maior número de participantes foi de mulheres ${ }^{(22,23)}$. Isso nos leva a inferir que as mulheres com DM, principalmente aquelas que são do lar, estão mais propensas às ações de educação em saúde, pois tem a oportunidade de deixar os afazeres domésticos para aprender a cuidar um pouco mais de si e lidar melhor com as limitações que a doença pode ocasionar.

Uma investigação realizada no Nepal envolvendo 132 individuos com DM, cujo objetivo foi compreender as barreiras para a educação em DM, mostrou que a idade avançada é um fator dificultador para a aprendizagem e mudança nos hábitos, fator esse relacionado com a deterioração da função cognitiva, sendo que os pacientes mais jovens demonstram ter maior motivação e adaptabilidade à doença ${ }^{(24)}$.

O baixo nível de escolaridade também é considerado um fator limitante ao acesso às informações, dificultando a compreensão frente às orientações recebidas, impactando negativamente no automanejo da doença ${ }^{(25)}$.

A avaliação realizada a partir da aplicação da escala OAD antes e após a participação dos sujeitos no programa educativo resultou em diferenças significativas para as dimensões: alimentação geral e específica, atividade física, monitorização da glicemia e cuidados com os pés.

Para a dimensão da alimentação geral observou-se diferença estatística no número de dias que os indivíduos relataram seguir uma dieta saudável e orientada por algum profissional da saúde ( $p=0,01 ; p<0,001$ respectivamente).

O assunto alimentação sempre foi motivo para questionamentos, troca de experiências e orientações entre os participantes. Essa condição é considerada de fundamental importância quando se trata da educação popular em saúde. onde os temas geradores do aprendizado surgem das necessidades individuais e coletivas da população(15)

É importante destacar que, no presente estudo, quando questionado sobre o número de dias em que o sujeito seguiu a orientação alimentar fornecida no programa, a resposta foi em média de dois dias antes do programa e 5,6 dias após o programa.

O estudo de validação do OAD no Brasil mostrou que 3,4 dias foi a média de seguimento da orientação alimentar entre os sujeitos, porém não houve intervenção educativa no mesmo(13).

Neste estudo observou-se que, dentre as três questões dessa dimensão, apenas a questão sobre o consumo de doces mostrou diferença estatisticamente significativa após o programa. As mudanças de comportamento em relação a seguimento de orientações nutricionais são fundamentais na avaliação de programas educativos em DM, pois se espera que o individuo tenha modificado seu conhecimento e incorporado ações que irão refletir potencialmente no seu controle metabólico ${ }^{(25)}$.

$\mathrm{Na}$ avaliação do item atividade física, houve diferença estatística significativa quanto ao número de dias que os indivíduos praticaram algum tipo de exercício físico, sem incluir suas atividades de trabalho, sendo observado um aumento de zero para 2,3 dias por semana ( $p<0,001)$.

A monitorização glicêmica também foi uma das variáveis que apresentou diferença estatística significativa, com maior correlação interitens ( $\alpha$ 0,94). Quando questionados sobre o número de dias da semana que avaliavam a glicemia capilar, a média de respostas passou de 1,3 dias no início para 3,1 dias ao final do programa ( $p<0,007)$.

Quando questionados em relação a quantas vezes realizavam a glicemia capilar segundo a recomendação do médico ou enfermeiro, a média iniciou em 0,7 dias, passando para 3,1 dias ao final da intervenção educativa $(p<0,001)$.

A monitorização glicêmica tem se mostrado como um dos mais importantes indicadores para o autocuidado e autocontrole do DM, pois é através da visualização de resultados que as pessoas com a doença podem tomar decisões e compreender que está acontecendo no seu metabolismo(2).

A aquisição do glicosímetros e insumos necessários para a realização dos testes foi fator dificultador no presente 
estudo, dado o perfil socioeconômico dos participantes. Assim, observou-se que os sujeitos passaram a comparecer com maior frequência ao serviço de saúde para a realização da glicemia capilar em horários variados, sempre acompanhados por seus respectivos diários de glicemia capilar, após o programa educativo.

Os dados de autocuidado com os pés mostraram diferenças significativas para o número de dias que os sujeitos examinaram seus pés e seus sapatos antes de calçá-los (valores de $\mathrm{p}<0,001$ para ambos). A melhora do autocuidado com os pés reforça a diferença estatística da presença de ressecamento da pele avaliada após o término do programa educativo $(p=0,041)$

Com relação às variáveis clínicas avaliadas, $80 \%$ dos investigados apresentaram Hipertensão Arterial, dados estes coincidentes aos encontrados na literatura(22,23). Estudos mostram que cerca de $40 \%$ das pessoas que fazem o diagnóstico de DM2 já são hipertensos. O acompanhamento dos níveis pressóricos é de extrema importância uma vez que ajudam a prevenir a doença cardiovascular (DCV) bem como a progressão da doença renal e da retinopatia diabética(2,25).

Estudo realizado durante vinte anos (1977 a 1997) em 23 centros clínicos do Reino Unido mostrou conclusivamente que as complicações do DM2, anteriormente muitas vezes consideradas como inevitável, poderiam ser reduzidas, melhorando os niveis de glicose no sangue e/ou controle da pressão arterial(25).

A medida da hemoglobina glicada é outro método que permite a avaliação do controle glicêmico em longo prazo, e deve ser solicitada rotineiramente a todos os pacientes com DM, em média, a cada 3 (três) meses(1,2,25).

Neste estudo constatou-se uma média inicial da HbAlC de $7,6 \%$, ou seja, bem acima dos valores preconizados, e média final de $6,6 \%$. Os outros exames laboratoriais avaliados (glicemia de jejum, triglicérides, colesterol total e HDL) apresentaram redução não significativa estatisticamente.

Sabe-se que mais de $50 \%$ das pessoas idosas com DM2 tem um ou mais fatores de risco para ulceração do pé. Assim, torna-se importante a avaliação da integridade cutaneomucosa para identificação precoce de úlceras dos membros inferiores, ou "pé diabético". Aproximadamente, 15\% das pessoas com DM no mundo serão afetadas por úlceras de extremidades inferiores, que, se não tratadas, podem levar à amputação e óbito(1,5,7).

O conhecimento da história do paciente associado a um exame minucioso dos pés são as chaves para a classificação do risco de ulceração e prevenção. O rastreamento para a polineuropatia diabética (PND) engloba identificar sinais e sintomas de neuropatia e vasculopatia periféricas ${ }^{(1,5,7)}$.

Neste estudo, a avaliação dos pés pela enfermeira, utilizando as recomendações do Ministério da Saúde(14), aconteceu em dois momentos, antes e após a participação no programa educativo.

Das características avaliadas neste estudo, apenas a variável 'ressecamento' apresentou significância estatística após o programa educativo ( $p=0,041)$, fator este, possivelmente relacionado à mudança de comportamento dos indivíduos em relação ao autocuidado.

A realização de testes pouco complexos como o exame físico dos pés e os testes de sensibilidade protetora pelos profissionais de saúde, especialmente aqueles que atuam na atenção primária, é de fácil acesso e execução.

Estudos indicam a importância do papel do enfermeiro da atenção primária e secundária nas ações que se referem ao rastreamento da doença, prevenção, classificação do risco e medidas de controle, e a presença de um profissional capacitado especificamente em gerontogeriatria para estimular as competências para 0 autocuidado dos idosos $(1,2,24)$

\section{Limitações do estudo}

Este estudo limita-se ao baixo número de participantes no programa educativo, o que não permite generalizar os resultados favoráveis obtidos com o desenvolvimento da estratégia utilizada.

\section{Contribuições do estudo para a prática}

O enfermeiro tem um papel crucial, na organização da assistência e na condução de processos educativos que confiram empoderamento e estimulem a incorporação das ações para o autocuidado. Assim, esta pesquisa permitiu em curto período de tempo, em um grupo pequeno, mudar concepções básicas sobre os cuidados com DM, e apontar uma medida mensurável pelo OAD para melhor avaliar o controle clínico dos diagnosticados.

\section{CONCLUSÃO}

Diante do exposto, observou-se que o programa educativo atendeu aos objetivos propostos, uma vez que permitiu a melhora na alimentação específica, atividade física, monitorização da glicemia e cuidados com os pés dos participantes, levando a inferir que a educação para o autocuidado do DM pode proporcionar redução do risco de complicações decorrentes da doença.

O estudo também demonstrou que os parâmetros clínicos e laboratoriais como HBAlc, pressão arterial, IMC lipidogramas merecem ser melhor investigados por um tempo maior de acompanhamento.

Outro aspecto importante a ser destacado é que a educação em saúde pode fornecer subsídios para que as 
pessoas que vivem com DM tenham maior autocuidado com a sua monitorização glicêmica e com os pés. Nesse sentido, acreditamos que o uso da escala QAD para avaliar as atividades de autocuidado com DM é pertinente para a mensuração de resultados após as atividades educativas realizadas.

Conclui-se, portanto que, programas de educação baseados no Mapa de Conversação em Diabetes® demonstram ser fundamentais para a capacitação da pessoa com DM no manejo da doença no sentido de prevenir suas complicações.

\section{Contribuição dos autores}

Os autores deste artigo Ohl RIB, Chavaglia SRR, Gomes JLGC, Freitas MAO e Gamba MA declaram que contribuíram com a concepção e projeto, análise e interpretação dos dados, redação do artigo, revisão crítica relevante do conteúdo intelectual e aprovação final da versão a ser publicada. 


\section{REFERÊNCIAS}

1. Sociedade Brasileira de Diabetes. Diretrizes da Sociedade Brasileira de Diabetes: 2015-2016. Diretrizes para educação do paciente com Diabetes Mellitus. Sociedade Brasileira de Diabetes. São Paulo: AC Farmacêutica. 2016; 223-229.

2. Brasil. Ministério da Saúde. Secretaria de Atenção à Saúde Departamento de Atenção Básica. Diretrizes para o cuidado das pessoas com doenças crônicas nas redes de atenção à saúde e nas linhas de cuidado prioritárias / Ministério da Saúde, Secretaria de Atenção à Saúde, Departamento de Atenção Básica. Brasilia: Ministério da Saúde;2013.

3. Ministério da Saúde (BR). Secretaria de Vigilância em Saúde. Departamento de Análise de Situação de Saúde. Plano de ações estratégicas para o enfrentamento das doenças crônicas não transmissiveis (DCNT) no Brasil 2011-2022 / Ministério da Saúde. Secretaria de Vigilância em Saúde. Departamento de Análise de Situação de Saúde. Brasília: Ministério da Saúde;2011.

4. Malta DC, Silva MMA, Moura L, Neto OLM. The implantation of the surveillance system for non-communicable diseases in Brazil, 2003 to 2015: successes and challenges. Rev Bras Epidemiol [Internet] 2017[cited 2017 Dec 1]; 20(4):66l-675 Available from: https://www. scielosp.org/pdf/rbepid/2017.v20n4/661-675/pt

5. Gomides DS, Villas-Boas LC, Coelho AC, Pace AE. Self-care of people with diabetes mellitus who have lower limb complications. Acta Paul Enferm [Internet]. 2013[cited 2016 Jan 28]:26(3):289-93. Available from: http://www.scielo.br/pdf/ape/v26n3/14.pdf

6. Borba AKOT, Marques APO, Leal MCC, Ramos RSPS. Educational practices for diabetes Mellitus: integrative literature review. Rev Gaúcha Enferm [Internet]. 2012 [cited 2015 Apr18];33(1):169-76. Available from: http://www.scielo.br/pdf/rgenf/v33nl/a22v33nl.pdf

7. Amaral MA, Amorim MMA, Torres HC, Abreu CRT. Educational workshop in primary health care for promoting self-care in diabetes mellitus. Rev. APS [Internet]. 2014[cited 2015 Jun 28]; 17(1): 58 64. Available from: https://aps.ufjf.emnuvens.com.br/aps/article/ view/1871/785

8. Brown MA, Davis CS. Group education tool: The Conversation Map® programme. Journal of Diabetes Nursing [Internet]. 2016[cited 2018 Mar 21];20(7):251-54. Available from: http://www. thejournalofdiabetesnursing.co.uk/media/content/_master/4681/ files/pdf/jdn20-7-25l-4.pdf

9. Chinenye S, Young EE. Diabetes conversation map in Nigeria: a new socioeducational tool in diabetes care. Indian $\mathrm{J}$ Endocr Metab [Internet]. 2013.[cited 2015 Dec 11];17(6):1009-11 Available from: http:// www.ncbi.nlm.nih.gov/pmc/articles/PMC3872677/

10. Chaves FF, Chaves FA, Cecilio SG, Amaral MA, Torres HC Conversation map on diabetes: education strategy in view of health professionals. REME - Rev Min Enferm [Internet]. 2015[cited 2015 Apr 11]; 19(4): 854-858. Available from: http://www.reme.org.br/artigo/ detalhes/1046

11. Li F, Yao P, Hsue C, Xu J, Lou O. Impact of "Conversation Maps" on diabetes distress and self-efficacy of Chinese adult patients with type 2 diabetes: a pilot study. Patient Prefer Adherence [Internet] 2016[cited 2016 Oct 13]; 10: 901-8. Available from: https://www.ncbi. nlm.nih.gov/pmc/articles/PMC4889097/pdf/ppa-10-901.pdf

12. Polit DF, Beck CT. Fundamentos de pesquisa em enfermagem: avaliação de evidências para a prática da enfermagem. 7. ed. Porto Alegre: Artmed, 2011.

13. Michels M J, Coral MHC, Sakae TM, Damas TB, Furlanetto LM Questionnaire of Diabetes Self-Care Activities: translation, crosscultural adaptation and evaluation of psychometric properties. Arq Bras Endocrinol [Internet]. 2010 [cited 2015 Jul 28]; 54(7):644-51 Available from: http://www.scielo.br/pdf/abem/v54n7/09.pdf

14. Torres HC, Pereira FRL, Alexandre LR. Evaluation of the educational practices in promoting self-management in type 2 diabetes mellitus. Rev Esc Enferm USP [Internet]. 2011 [cited 2016 Jun 12] 45(5):1077-82. Available from: http://dx.doi.org/10.1590/S008062342011000500007
15. Goes TM, Polaro SHI, Gonçalves LHT. Cultivo do bem viver das pessoas idosas e tecnologia cuidativo-educacional de enfermagem. Enferm Foco [Internet]. 2016 [cited 2019 Apr 09]; 7 (2): 47-51 Available from: http://revista.cofen.gov.br/index.php/enfermagem/article/ view/794/319

16. Nunes JM, Oliveira EN, Machado MFAS, Costa PNP, Vieira NFC. Being a woman and taking part in a community health educational group: motivations and expectations. Rev enferm UERJ [Internet]. 2014[cited 2015 Nov 19]; (1):123-8. Available from: http://bmchealthservres. biomedcentral.com/articles/10.1186/1472-6963-12-213

17. Steinsbekk A, Rygg L, Lisulo M, Rise MB, Fretheim A. Group based diabetes self-management education compared to routine treatment for people with type 2 diabetes mellitus. A systematic review with meta-analysis. BMC Health Services Research [Internet]. 2012[cited 2015 Oct 08]; 12(213):1-19 Available from: http://bmchealthservres. biomedcentral.com/articles/10.1186/1472-6963-12-213

\section{Effectiveness of Interactive Self-Management}

Interventions in Individuals With Poorly

Controlled Type 2 Diabetes: A Meta-Analysis

of Randomized Controlled Trials

18. Cheng L, Sit JWH, Choi K, Chair S, Li X, He XL. Effectiveness of Interactive Self Management interventions in individuals with poorly controlled type 2 diabetes: a meta analysis of randomized controlled trials. Worldviews on Evidence-Based Nursing [Internet] 2017[cited 2018 Apr 24]; 14:1, 65-73. Available from: https://sigmapubs. onlinelibrary.wiley.com/doi/pdf/10.1111/wvn.12191

19. Reaney M, Zorzo EG, Golay A, Hermanns N, Cleall S, Petzinger U et al. Impact of Conversation Map ${ }^{T M}$ Education Tools Versus Regular Care on Diabetes-Related Knowledge of People with Type 2 Diabetes: A Randomized, Controlled Study. Diabetes Spectrum [Internet]. 2013[cited 2015 Apr 3]; 26(4):236-45 Available from: http://spectrum. diabetesjournals.org/content/26/4/236

20. El Hait SS, Chaar B, Mclachlan AJ, Overland J, Basheti IA. Subjective assessment to an educational tool used for the Arabic population with diabetes in Australia. Jordan Journal of Pharmaceutical Sciences [Internet]. 2014[cited 2015 May 16]; 7(3): 180-90. Available from: https:// www.researchgate.net/publication/264232907

21. Penalba M, Moreno L, Cobo A, Reviriego J, Rodriguez A, Cleall S et al. Impact of "Conversation Map ${ }^{\text {TM" }}$ tools on understanding of diabetes by Spanish patients with type 2 diabetes mellitus: A randomized comparative study. Endocrinología y Nutrición [Internet]. 2014[cited 2015 Mar 3]; 61(10):505-15 Available from: http://www.ncbi.nlm.nih.gov/ pubmed/25085598

22. Lessmann JC, Silva DMGV, Nassar SM. Women with type 2 diabetes mellitus: sociodemographic profile, biometrics and health. Acta Pau Enferm [Internet]. 2012[cited 2015 Dec 17];25(1):81-6. Available from: http://www.scielo.br/pdf/ape/v25nspel/pt_13.pdf

23. Ahmed MM, El Degwy HM, Ali MI, Hegazy NH. The effect of educational intervention on knowledge, attitude and glycemic contro in patients with type 2 diabetes mellitus. Int $\mathrm{J}$ Community Med Public Health[Internet]. 2015[cited 2015 Nov 29];2(3):302-307 Available from: http://www.ijcmph.com/index.php/ijcmph/article/view/970/839

24. Shrestha N, Yadav SB, Joshi AM, Patel BDP, Shrestha, Bharkher DL. Diabetes Knowledge and Associated Factors among Diabetes Patients in Central Nepal. International Journal of Collaborative Research on Internal Medicine \& Public Health [Internet]. 2015[cited 2015 Feb 8] 7(5):82-91 Available from: http://www.iomcworld.com/ijcrimph/files/ v07-n05-01.pdf

25. Arrelias CC, Faria HT, Teixeira CR, Santos MA, Zanetti ML. Adherence to diabetes mellitus treatment and sociodemographic, clinical and metabolic control variables. Acta Paul Enferm [Internet]. 2015[cited 2015 Mar 21]; 28(4):315-22. Available from: http://www.scielo.br/ readcube/epdf.php?doi=10.1590/1982-01942015000548pid=S010321002015000400005 \& pdf_path=ape/v28n4/1982-0194ape-28-04-0315.pdfElang=pt 